\title{
Spin transport in cold Fermi gases: A pseudogap interpretation of spin diffusion experiments at unitarity
}

\author{
Dan Wulin, ${ }^{1}$ Hao Guo, ${ }^{2}$ Chih-Chun Chien, ${ }^{3}$ and K. Levin ${ }^{1}$ \\ ${ }^{1}$ James Franck Institute and Department of Physics, University of Chicago, Chicago, Illinois 60637, USA \\ ${ }^{2}$ Department of Physics, University of Hong Kong, Hong Kong, China \\ ${ }^{3}$ Theoretical Division, Los Alamos National Laboratory, MS B213, Los Alamos, New Mexico 87545, USA
}

(Received 10 February 2011; published 6 June 2011)

\begin{abstract}
We address recent spin transport experiments in ultracold unitary Fermi gases. We provide a theoretical understanding for how the measured temperature dependence of the spin diffusivity at low $T$ can disagree with the expected behavior of a Fermi liquid (FL), while the spin susceptiblity, following the experimental protocols, is consistent with a FL picture. We show that the experimental protocols for extracting the spin susceptibility implicitly reflect a FL viewpoint; relaxing this leads to consistency within but not proof of a pseudogap-based theory. Our transport calculations yield insight into the observed suppression of the spin diffusion constant at lower $T$.
\end{abstract}

DOI: 10.1103/PhysRevA.83.061601

PACS number(s): 03.75.Ss, 67.10.Jn, 67.85.De

Recent measurements associated with mass transport [1] and spin transport [2] in ultracold Fermi gases and superfluids are of great interest principally because they provide detailed information about the excitation spectrum, thereby strongly constraining microscopic theories. Equally important are their widespread implications for a host of different strongly correlated systems, ranging from guark-gluon plasmas to high $T_{c}$ cuprates. A commonality of these materials is the short mean free paths that lead to "near-perfect fluidity" [1], "bad metallicity" [3], and now bad spin conductivity [2]. The latter are Fermi-gas-based materials with small spin diffusivities $D_{s}$ that approach the quantum limited value $\hbar / m$ as the temperature is lowered. Importantly, the nature of the excitations in these Fermi gases is currently under debate [4,5] and a controversy has emerged as to whether the associated normal state of these superfluids is a Fermi liquid (FL) or whether it contains an excitation (pseudo)gap.

The goal of this Rapid Communication is to address the recent spin transport experiments by Sommer et al. [2] that measure the spin diffusivity $D_{s}$, the spin diffusion-associated lifetime $\tau_{s}(T)$ (which enters into the spin conductivity $\sigma_{s}$ ), and thereby deduce the spin susceptibility $\chi_{s}=\sigma_{s} / D_{s}$. There are mixed features that have been experimentally reported for these transport variables, both consistent and inconsistent with FL theory, so that a single self-consistent description of the phenomenology was apparently not possible. The way in which the present Rapid Communication resolves this discrepancy is to present a careful calculation of the spin conductivity and susceptibility. When the pseudogap is included in the transport properties, one recovers a consistent interpretation of both $\chi_{s}$ and $D_{s}$, in contrast to the FL approach, which leads to a mixed interpretation. We show that the quantity $\sigma_{s}$ can only be assumed (as in Ref. [2]) to be simply proportional to the relaxation time within a FL description. In the presence of a pseudogap, the carrier number that enters into the spin conductivity is temperature dependent. This is physically reasonable, since a gap in the fermionic excitation spectrum above the transition at $T_{c}$ in the unitary gas must reduce the number of carriers in the normal state, leading to a temperature-dependent carrier number.
Before embarking on the detailed calculations, it is useful to list some of the key findings and results. The spin susceptibility can be related to the spin conductivity as $\chi_{s} \equiv \sigma_{s} / D_{s}$, where the left-hand side, in a FL picture, can be written as

$$
\chi_{s}^{\mathrm{FL}} \equiv \frac{n}{m} \tau_{s}(T) / D_{s}, \quad \frac{n}{m}=\text { const. }
$$

Here $n / m$ corresponds to the usual transport definition for the carrier number divided by (bare) mass, e.g., $n / m=$ $(2 / 3) \sum\left(\partial \xi_{\mathbf{p}} / \partial \mathbf{p}\right)^{2}\left(-\partial f\left(\xi_{\mathbf{p}}\right) / \partial \xi_{\mathbf{p}}\right)$ for quasiparticle dispersion $\xi_{\mathbf{p}}$, where $f$ is the Fermi function. By contrast, in our non-FL pseudogap model, we will show using spin-spin correlation functions that the spin conductivity is of the general form

$$
\sigma_{s}^{\mathrm{pg}}=\left[\frac{n}{m}(T)\right]_{\mathrm{eff}} \tau_{s}(T)=\frac{2}{3} \tau_{s} \sum_{\mathbf{p}} \mathbf{v}_{\mathbf{p}}^{2}\left(-\frac{\partial f\left(E_{\mathbf{p}}\right)}{\partial E_{\mathbf{p}}}\right) .
$$

Here $E_{\mathbf{p}}=\sqrt{\xi_{\mathbf{p}}^{2}+\Delta^{2}}$, where $\Delta$ is the pairing gap, and $\mathbf{v}_{\mathbf{p}}=$ $\partial E_{\mathbf{p}} / \partial \mathbf{p}$. The spin susceptibility in the presence of a pseudogap (derived from the spin-spin correlation functions) is

$$
\chi_{s}=\sum_{\mathbf{p}}\left(-\frac{\partial f}{\partial E_{\mathbf{p}}}\right) .
$$

To obtain the above results, we begin with the general spin-spin correlation function called $Q_{00}^{s}(\mathbf{q}, \omega)$. It follows from Eq. (6) and Ref. [6] that the spin conductivity is $\sigma_{s}=-\lim _{\omega \rightarrow 0} \lim _{\mathbf{q} \rightarrow \mathbf{0}}\left(\omega / \mathbf{q}^{2}\right) \operatorname{Im} Q_{00}^{s}(\mathbf{q}, \omega) / \pi$. Any consistent theory of the spin transport must be compatible with the $f$-sum rule

$$
\int_{-\infty}^{\infty} d \omega \omega \chi^{\prime \prime}(\mathbf{q}, \omega) / \pi=n \mathbf{q}^{2} / m,
$$

where $\chi^{\prime \prime}(\mathbf{q}, \omega)=-\operatorname{Im} \mathbf{Q}_{00}^{s} / \pi$. We have analytically verified this to be satisfied in the approach below. From the spinspin correlation function, one derives the spin susceptiblity $\chi_{s}=\int_{-\infty}^{\infty} d \omega \chi^{\prime \prime}(\mathbf{q}, \omega) / \pi \omega$ which, in the present case, leads to Eq. (3).

The physics of transport in the unitary gas is complicated because the pseudogap in the normal state $[7,8]$ requires that both fermions and fermionic pairs be considered. In recent 
papers [6,9], we have included both types of quasiparticles in addressing the observed anomalously low shear viscosity [1] both above and below $T_{c}$, as well as magnetic and nonmagnetic Bragg scattering [6]. While previous studies of spin transport in the cold Fermi gases have employed, for example, quantum Monte Carlo calculations, Boltzmann transport, and variational approaches [10,11], we choose to use the Kubo formalism that more readily addresses conservation laws and sum rules. The underlying theory is a BCS-BEC crossover theory in a $t$-matrix formulation [8]. At $T=0$, the system is in the BCS ground state where there are only superconducting pairs characterized by the gap $\Delta_{\text {sc }}$. For temperatures $0<T \leqslant T_{c}$, there is a comixture of superconducting pairs, noncondensed pairs characterized by the gap $\Delta_{\mathrm{pg}}$, and fermionic quasiparticle excitations.

Essential to satisfying conservation laws and sum rules is the incorporation of Ward identities and collective mode physics. These issues are addressed in Refs. [6] and [12]. The presence of both condensed and noncondensed pairs in transport can be accommodated with the introduction of the usual Maki-Thompson (MT) and Aslamazov-Larkin (AL) diagrams. These are incorporated in a fashion that is demonstrably consistent with gauge invariance. A different diagram subset necessarily appears in the spin response, as compared with the mass transport (or electromagnetic response); in the former, the AL diagrams are not present nor do collective mode effects enter. The fermionic self-energy that we obtain has, above $T_{c}$, been widely used in pseudogap theories (such as those of Senthil and Lee [13] and others [14]). It is

$$
\Sigma\left(\mathbf{p}, i \omega_{n}\right)=-i \gamma+\frac{\Delta_{\mathrm{pg}}^{2}}{i \omega_{n}+\xi_{\mathbf{p}}+i \gamma}+\frac{\Delta_{\mathrm{sc}}^{2}}{i \omega_{n}+\xi_{\mathbf{p}}},
$$

where $\gamma$ is the fermionic inverse lifetime associated with noncondensed pairs. This lifetime is due to fermion-pair interconversion and the general form for $\Sigma$ in Eq. (5) has been applied in both experimental [15] and theoretical [16] rf studies of cold Fermi gases. In the weak dissipation limit where $\gamma$ is small, there is little distinction between condensed and noncondensed pairs, whereas in the strong dissipation limit the distinction is significant. In this Rapid Communication, we find that these two approaches tend to converge for $s$-wave pairing and for the lifetime parameters obtained from rf experiments. Even though it is likely that $\tau_{s}$ is smallest at unitarity, its effects on $\sigma_{s}$ do not overwhelm the strong reduction in the carrier number due to the excitation gap; the behavior is very different for $d$-wave pairing in the cuprates.

We write the key equations first for the dc spin conductivity in the more general strong dissipation limit and second for the the spin-spin correlation function $Q_{00}^{s}(\mathbf{q}, \omega)$ at general $(\mathbf{q}, \omega)$, but in the limit in which the noncondensed pair lifetime is relatively long:

$$
\begin{aligned}
\sigma_{s}=-\lim _{\omega \rightarrow 0} \lim _{q \rightarrow 0} & \frac{1}{6 m^{2} \omega} \operatorname{Im} \sum_{P} \mathbf{p}^{2}\left[G_{P^{+}} G_{P^{-}}-F_{\mathrm{Sc}, P^{+}} F_{\mathrm{Sc}, P^{-}}-F_{\mathrm{pg}, \mathrm{P}^{+}} F_{\mathrm{pg}, \mathrm{P}^{-}}\right]_{i \Omega_{l} \rightarrow \omega^{+}}, \\
Q_{00}^{s}(\mathbf{q}, \omega)= & \sum_{\mathbf{p}}\left[\frac{E_{\mathbf{p}^{+}}+E_{\mathbf{p}^{-}}}{E_{\mathbf{p}^{+}} E_{\mathbf{p}^{-}}} \frac{E_{\mathbf{p}^{+}} E_{\mathbf{p}^{-}}-\xi_{\mathbf{p}^{+}} \xi_{\mathbf{p}^{-}}-\Delta^{2}}{\omega^{2}-\left(E_{\mathbf{p}^{+}}+E_{\mathbf{p}^{-}}\right)^{2}}\left[1-f\left(E_{\mathbf{p}^{+}}\right)-f\left(E_{\mathbf{p}^{-}}\right)\right]\right. \\
& \left.-\frac{E_{\mathbf{p}^{+}}-E_{\mathbf{p}^{-}}}{E_{\mathbf{p}^{+}} E_{\mathbf{p}^{-}}} \frac{E_{\mathbf{p}^{+}} E_{\mathbf{p}^{-}}+\xi_{\mathbf{p}^{+}} \xi_{\mathbf{p}^{-}}+\Delta^{2}}{\omega^{2}-\left(E_{\mathbf{p}^{+}}-E_{\mathbf{p}^{-}}\right)^{2}}\left[f\left(E_{\mathbf{p}^{+}}\right)-f\left(E_{\mathbf{p}^{-}}\right)\right]\right],
\end{aligned}
$$

where $P^{+}=\left(\mathbf{p}+\frac{\mathbf{q}}{2}, i \omega_{n}+i \Omega_{l}\right), P^{-}=\left(\mathbf{p}-\frac{\mathbf{q}}{2}, i \omega_{n}\right), \mathbf{p}^{ \pm}=$ $\mathbf{p} \pm \mathbf{q} / 2$, and $\omega^{+}=\omega+i 0^{+}$. The quantity $\omega_{n}\left(\Omega_{l}\right)$ is a fermionic (bosonic) Matsubara frequency. Moreover, $\Delta=$ $\sqrt{\Delta_{\mathrm{sc}}^{2}+\Delta_{\mathrm{pg}}^{2}}$ appears in the excitation energy $E_{\mathbf{p}}$. Here
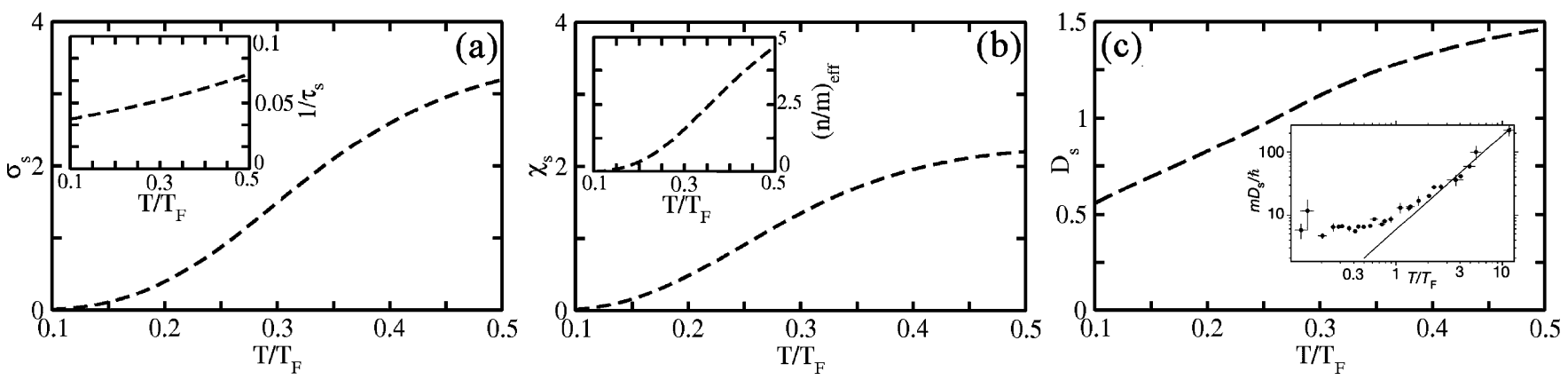

FIG. 1. Transport properties in the presence of a pseudogap. Unless otherwise noted, results are normalized by their values at $T_{c}=0.26 T_{F}$. The plots correspond to $0.1 T_{F}<T<0.5 T_{F}$, where the rf-lifetime data are available. (a) The spin conductivity $\sigma_{s}$. The inset shows the quasiparticle inverse lifetime $\tau_{s}^{-1}$ in units $E_{f} / \hbar$. (b) The spin susceptibility $\chi_{s}$. The inset displays the temperature-dependent effective carrier number $(n / m)_{\text {eff. }}$ (c) The spin diffusivity $D_{s}$ with experimental data [2] in the inset. 
$G=G(\Sigma)$ is the dressed Green's function and one can interpret $F_{\text {sc }}$ as the usual Gor'kov Green's function reflecting superconducting order; however, there must also be a counterpart $F_{\mathrm{pg}}$ (discussed in detail elsewhere [17] that reflects noncondensed pairs). Interestingly, in the weak dissipation limit, the spin transport correlation functions depend only on the total pairing gap $\Delta$. Moreover, when pseudogap effects are dropped, these equations reduce to their usual BCS counterparts.

We turn now to a comparison between theory and experiment. In the inset of Fig. 1(a), the values of $\tau_{s}^{-1}$ inferred from rf experiments are plotted in units of $E_{F} / \hbar$ [9]. Given the experimental definition [2] of spin conductivity as $\sigma_{s}=\frac{n}{m \Gamma_{\mathrm{sd}}}$, we associate $\Gamma_{\mathrm{sd}}$, as measured experimentally, with $\tau_{s}^{-1}$. Another important association via the microscopic Kubo calculation leads to an identification between the spin-drag lifetime $\tau_{s}$ and the quasiparticle lifetime $\gamma^{-1}$; spin and "charge" or mass are carried by the same quasiparticles. This identification was also observed in previous Kubo calculations of spin diffusion in ${ }^{3} \mathrm{He}[18]$.

It can be seen from the inset that the lifetime increases for lower temperatures since interconversion processes cease when noncondensed bosons disappear; in this way fermions become long lived. That the interconversion between fermions and bosons is the physical origin of this lifetime suggests that $\tau_{s}$ is smallest for the unitary gas [17], where the number of bosonic pairs and fermions is roughly comparable. This appears consistent with the findings in Ref. [2]. Importantly, both the magnitude and the temperature dependences of the inverse lifetimes $\left(\Gamma_{\mathrm{sd}}\right.$ and $\left.\tau_{s}^{-1}\right)$ are in rough agreement.

The main body of Fig. 1(a) presents a plot of the spin conductivity $\sigma_{s}$ as deduced from Eq. (7) and independently checked via the strong dissipation Green's function form of Eq. (6). Importantly, even though the lifetime is longer as temperature is lowered, because of a gap in the fermionic excitation spectrum the conductivity becomes very small at low $T$. This reflects the effective carrier number as defined in Eq. (2).

The calculated spin susceptibility $\chi_{s}$ is shown in the main body of Fig. 1(b). This has the expected temperature dependence associated with a pseudogap. Plotted in the inset of Fig. 1(b) is the effective carrier number $(n / m)_{\text {eff }}[$ Eq. (2)] as a function of temperature. As also seen in plots of $\sigma_{s}$, the quantity $(n / m)_{\text {eff }}$ vanishes with decreasing temperature due to the pairing gap. Finally, in Fig. 1(c), we plot the microscopically computed spin diffusivity $D_{s}=\sigma_{s} / \chi_{s}$ derived from $\chi_{s}$ and $\sigma_{s}$. Importantly, we find the spin diffusivity is suppressed at low temperatures, reflecting the suppression in the spin conductivity $\sigma_{s}$ as a result of the reduced number of carriers. The experimentally measured $D_{s}$ is shown in the inset of Fig. 1(c). As in the theory, $D_{s}$ is found to decrease at low $T$. It appears [2] to approach the universal quantum limit $\hbar / \mathrm{m}$.

We turn now to Fig. 2, which plots the spin susceptibility derived following the protocol in Ref. [2] [Eq. (1)]. We use the superscript "FL" to emphasize that this analysis builds in a FL interpretation by assuming that the carrier number in the spin conductivity is a constant in temperature and that no excitation gap is present. In this case, one can see that the spin susceptibility increases for low temperature. This is distinctly different from what is plotted in Fig. 1(b) reflecting

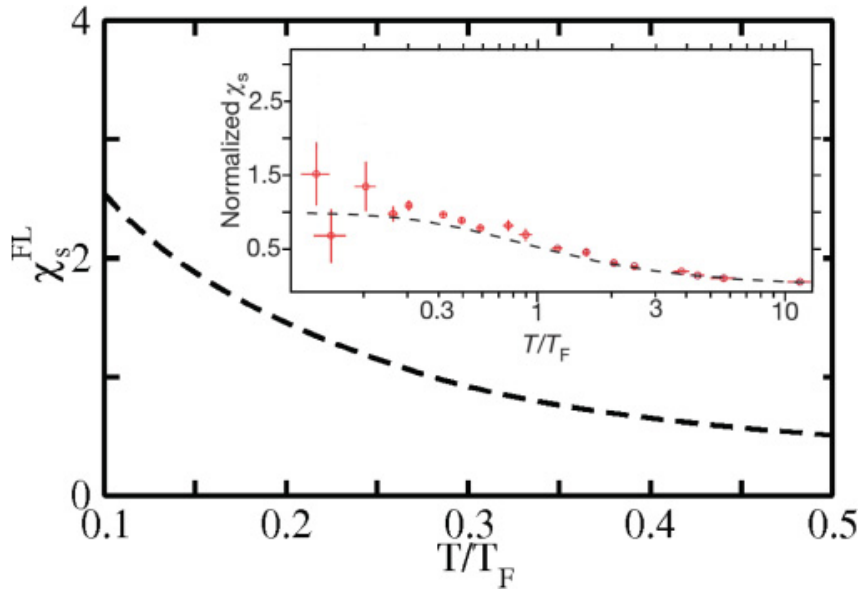

FIG. 2. (Color online) Plot of the theoretically deduced Fermi-liquid spin susceptiblity $\chi_{s}^{\mathrm{FL}} \equiv \frac{n}{m} \tau_{s}(T) / D_{s}$ based on the experimental protocol assuming that $n / m$ is constant in temperature (and normalized by the $T_{c}$ value). The inset plots experimental [2] $\chi_{s}^{\mathrm{FL}}$, in units $3 n / 2 E_{F}$.

the presence of a pseudogap. Nevertheless, it can be seen following this protocol that $\chi_{s}^{\mathrm{FL}}$ exhibits a $T$ dependence similar to that reported experimentally; the low $T$ upturn is more marked in the calculation. By adjusting $\tau_{s}(T)$ to be more in line with the counterpart experiment, one can arrive at better agreement between this $\chi_{s}^{\mathrm{FL}}$ and the data. We stress, however, that Fig. 2 should not be assumed to support this FL-based interpretation,- but rather to establish that if the behavior for $\sigma_{s}$ is assumed to be FL like, the computed $\chi_{s}$ will also be so, as in the experiment of Ref. [2].

In this Rapid Communication, we have not proven one way or the other whether there is strong evidence for a pseudogap associated with the experimental results presented in Ref. [2]. What we have established is that the inferences from the experimentally obtained spin susceptibility [2], which are used to support FL theory, implicitly assume the absence of a gap. They are based on Eq. (1), which presumes a FL-like behavior, so that one cannot make independent inferences about the absence of a pseudogap. Other evidence against a pseudogap based on thermodynamics of a balanced unitary gas [4] can be countered by noting that the same thermodynamic power fits can be found for a non-FL (for example, in BCS theory below $T_{c}$ ). Recent experiments [5] that address the low $T$ normal phase associated with a Fermi gas of arbitrary imbalance are also problematic since the only theoretical phase diagram [19] (of which we aware) addressing where pseudogap and FL phases are stable predicts this low $T$ phase should be a FL, as is observed [5].

A suggestion for establishing how to rule in or out a pseudogap in the normal phase of the unitary gas is discussed in Ref. [20]. Essential is that one first confirms the known characteristics of the superfluid phase, such as a suppressed spin susceptibility and entropy, and then establish that these features persist somewhat above the transition. These observations are the counterpart of those first applied to the cuprates.

Of great interest is the relation between spin and mass transport. Because spin and "charge" or mass are carried by the same quasiparticles, even in the presence of a pseudogap [6], 
spin and mass transport behave similarly leading to "nearperfect fluidity," bad metallicity [3], and bad spin conductivity, which has been discussed here. Indeed, the analysis applied in this paper was used to anticipate that the anomalously low shear viscosity [9] of the normal state should persist down to $T \approx 0$, as now observed [1]. As in Ref. [21], we find the excitation gap, which suppresses the carrier number [Eq. (2)] is responsible for this behavior. It would similarly explain bad metallicity [3] in the pseudogapped high $T_{c}$ superconductors [17]. It is striking that recent experiments from the high $T_{c}$ community [22] have tended to focus on the temperature dependence of the effective number of carriers (in the presence of a pseudogap) and argue that it will affect transport "because $n_{\text {eff }}$ may be changing with $T$." These commonalities highlight the importance of the ultracold gases as powerful simulation tools for a wide class of condensed matter systems.

This work is supported by NSF-MRSEC Grant No. 0820054. C.C.C. acknowledges the support of the US Department of Energy through the LANL/LDRD Program.
[1] C. Cao, E. Elliot, J. Joseph, H. Wu, J. Petricka, T. Schafer, and J. E. Thomas, Science 331, 58 (2011).

[2] A. Sommer, M. Ku, G. Roati, and M. W. Zwierlein, Nature 472, 201 (2011)

[3] V. J. Emery and S. A. Kivelson, Nature (London) 374, 434 (1995).

[4] S. Nascimbene, N. Navon, K. J. Jiang, F. Chevy, and C. Salomon, Nature (London) 463, 1057 (2010).

[5] S. Nascimbene et al., e-print arXiv:1012.4664.

[6] H. Guo, C.-C. Chien, and K. Levin, Phys. Rev. Lett. 105, 120401 (2010).

[7] J. Stajic, J. N. Milstein, Q. J. Chen, M. L. Chiofalo, M. J. Holland, and K. Levin, Phys. Rev. A 69, 063610 (2004).

[8] Q. J. Chen, J. Stajic, S. N. Tan, and K. Levin, Phys. Rep. 412, 1 (2005).

[9] H. Guo, D. Wulin, C.-C. Chien, and K. Levin, e-print arXiv:1008.0423.

[10] M. M. Parish and D. A. Huse, Phys. Rev. A 80, 063605 (2009).

[11] G. M. Bruin, New J. Phys. 13, 035005 (2011).
[12] I. Kosztin, Q. J. Chen, Y.-J. Kao, and K. Levin, Phys. Rev. B 61, 11662 (2000).

[13] T. Senthil and P. Lee, Phys. Rev. B 79, 245116 (2009).

[14] A. Levchenko, T. Micklitz, M. R. Norman, and I. Paul, Phys. Rev. B 82, 060502(R) (2010).

[15] J. T. Stewart, J. P. Gaebler, and D. S. Jin, Nature (London) 454 744 (2008).

[16] Q. Chen, Y. He, C.-C. Chien, and K. Levin, Rep. Prog. Phys. 72 (2010).

[17] H. Guo, D. Wulin, C.-C. Chien, and K. Levin, e-print arXiv:1009.4678.

[18] M. A. Shahzamanian, J. Low Temp. Phys. 22, 27 (1976).

[19] C.-C. Chien, Q. J. Chen, Y. He, and K. Levin, Phys. Rev. Lett. 98, 110404 (2007).

[20] C.-C. Chien and K. Levin, Phys. Rev. A 82, 013603 (2010).

[21] G. M. Bruun and H. Smith, Phys. Rev. A 75, 043612 (2007).

[22] Y. S. Lee, K. Segawa, Z. Q. Li, W. J. Padilla, M. Dumm, S. V. Dordevic, C. C. Homes, Y. Ando, and D. N. Basov, Phys. Rev. B 72, 054529 (2005). 